\title{
CAPACIDAD DE INNOVACIÓN Y GESTIÓN DEL CONOCIMIENTO EN UNA INSTITUCIÓN DE EDUCACIÓN SUPERIOR
}

\author{
Capacity of Innovation and Knowledge Management in an \\ Institution of Higher Education
}

\author{
IGNACIO CONSUEGRA ARIZA \\ CAROLINA ARIZA BETHANCOURT \\ KARELIS BARRIOS HERNÁNDEZ
}

\begin{abstract}
RESUMEN
Este capítulo analiza la gestión del conocimiento y la capacidad de innovación en una institución de educación superior (IES) de Barranquilla. La investigación es relevante en la actualidad porque desarrolla aportes valiosos en el campo de estudio, del cual existen escasas evidencias científicas. Desde el punto de vista teórico, se aborda la gestión del conocimiento y capacidad de innovación en general en las organizaciones, para luego realizar un abordaje desde la visión de la IES. Se trata de un estudio descriptivo y exploratorio. La fuente primaria de recogida de datos, fue un cuestionario conformado por 15 ítems, medido en una escala Likert. Por último, se presentan los resultados obtenidos y principales conclusiones del estudio que dan evidencia de las implicaciones de la gestión del conocimiento en el desarrollo de la capacidad de innovación y, su efecto en la IES objeto de estudio.
\end{abstract}

Palabras clave: Gestión del conocimiento, capacidad de innovación, Ba, institución de educación superior.

\begin{abstract}
This chapter analyze of knowledge management and innovation capacity in a higher education institution (HEI) in Barranquilla. The research is relevant today, because it develops valuable contributions in the field of study because there is little scientific evidence. From a theoretical point of view, the management of knowledge and innovation capacity in general in the organizations is approached, and then an approach from the vision of the HEl. This is descriptive and exploratory study. The primary source of data collection was a questionnaire made up of 15 items, measured on a Likert scale. Finally, we present the results obtained and main conclusions of the study that give evidence of the implications of the knowledge management in the development of innovation capacity and its effect in the HEl object of study.
\end{abstract}

Keywords: Knowledge management, innovation capacity, Ba, higher education institution. 
GESTIÓN DEL CONOCIMIENTO Y CAPACIDAD DE INNOVACIÓN

Modelos, Sistemas y Aplicaciones

\section{INTRODUCCIÓN}

En un mundo de cambios implacables, donde las organizaciones constantemente experimentan transformaciones sustanciales en su estrategia, debido a entornos contingentes y competitivos que le rodean, la innovación, el conocimiento, la ciencia y la tecnología, desarrollan un papel fundamental, en el cual se hace necesario que las organizaciones gestionen su conocimiento, con el fin de desarrollar capacidades, especialmente la de innovación, de tal manera, que le permita adaptarse rápida y repetidamente a los cambios del entorno (Consuegra \& Ariza, 2016).

Por una parte, la gestión del conocimiento hace referencia a la administración de los procesos de creación, difusión y utilización del conocimiento mediante la fusión de tecnologías, estructuras organizativas y personas para crear aprendizaje valioso, solucionar problemas y tomar decisiones en una organización. Por otra parte, Teece, Pisano \& Shuen (1997) referencian la capacidad de innovación, como una capacidad dinámica, en la medida que se desarrolla como la habilidad para transformar continuamente el conocimiento y las ideas, en nuevos productos, procesos y sistemas, que benefician a la organización (Lawson \& Samson, 2001; Consuegra \& Ariza, 2016).

En este sentido, en países desarrollados son frecuentes las innovaciones generadas en los parques tecnológicos, donde empresas, universidades y otras organizaciones trabajan colaborativamente (Casallas, Plata \& Pineda, 2011; Morales et al., 2012), con el propósito de generar nuevo conocimiento e impactar de manera positiva en la sociedad. Acorde con lo anterior, es evidente la necesidad en países como Colombia, que se aúnen esfuerzos para gestionarlo y desarrollar la capacidad de innovación a partir de la interacción de las universidades con su entorno.

Es por eso que en los últimos años, las universidades en Barranquilla, han tenido la intención de definir e implementar estrategias que promuevan su capacidad de innovación, de tal manera que impacten no solo al interior de la organización, sino en el medio en el que desarrolla sus actividades (Consuegra \& Ariza, 2016). Para esto, se hace necesario identificar y conocer dicha capacidad, y su repercusión con el sector externo, lo que 
en consecuencia la ha llevado a comenzar a trabajar con la alianza Universidad-Empresa-Estado (Sábato \& Botana, 1968).

Esta tríada, busca aproximar a profesores investigadores con la realidad del entorno y con los requerimientos específicos de los diferentes sectores productivos de la sociedad; de esta manera, hallar por medio de sus proyectos articulados de investigación, soluciones prácticas e innovadoras para el entorno (Ramírez \& García, 2010; Consuegra \& Ariza, 2016).

Es así como este capítulo aborda un análisis de forma sistémica de la gestión del conocimiento y la capacidad de innovación de una universidad en Barranquilla, a partir de la necesidad imperante que las instituciones de educación superior (IES) sean fuente de generación de valioso conocimiento y de innovaciones que respondan a las necesidades propias de la sociedad.

\section{GESTIÓN DEL CONOCIMIENTO EN LAS ORGANIZACIONES}

En el contexto actual, los cambios presentados por el entorno han transformado la realidad de las organizaciones, poniendo de manifiesto la importancia de la gestión del conocimiento en la generación de valor para la creación de ventaja competitiva sostenible en el tiempo y el desarrollo de innovaciones que respondan a las necesidades del mercado.

Es así como la gerencia de las organizaciones ha evolucionado, de tal manera que para alcanzar mejores resultados en la gestión, se basen en el conocimiento, el aprendizaje y la innovación. Para Nonaka y Takeuchi (1995), el éxito las empresas solo es alcanzado en la medida que permanentemente creen nuevo conocimiento, lo difundan por toda la organización y lo incorporen rápidamente a nuevas tecnologías y productos.

Para gestionar el conocimiento, se requiere de un espacio compartido que involucre a las personas, los procesos, la estructura interna y la tecnología (Misra, 2007). Por eso, gestionarlo no es igual a la tecnología; para que el proceso se desarrolle de manera efectiva se requiere de los cuatro elementos. 
GESTIÓN DEL CONOCIMIENTO Y CAPACIDAD DE INNOVACIÓN

Modelos, Sistemas y Aplicaciones

\section{1. $\mathrm{Ba}$}

El Ba es la base fundamental para la gestión del conocimiento. Consiste en un espacio en el cual este es creado, explorado y explotado en la organización, constituyendo una fuente de ventaja competitiva, clave para la capacidad de innovación (Nonaka \& Takeuchi, 1995; Bueno \& Morcillo, 1997; Bueno, et al., 2006; Consuegra \& Ariza, 2016). El concepto de Ba es originario de Japón, propuesto en un primer momento por Nishida (1921) y posteriormente desarrollado por Shimizu (1995), quien lo refiere como un espacio (físico o intangible), intencional espontáneo, en el cual el conocimiento es creado, compartido y utilizado de diferentes formas y maneras.

En ese sentido, Nonaka \& Konno (1998) definen al Ba como un espacio físico, mental y virtual, o cualquiera de sus combinaciones, pensado para la promoción de relaciones emergentes y de ideas diferentes, desarrollado por personas y equipos de trabajo que se distinguen por sus resultados en la organización.

Estos espacios se convierten en apoyo fundamental para la toma de decisiones de la organización, ya que constituyen una ayuda para la creación y desarrollo de soluciones efectivas sobre las debilidades y amenazas que presenta el entorno de la organización (Acosta, et al., 2014).

Por otra parte, las interacciones e interrelaciones entre los diferentes miembros de la organización, son esenciales para el desarrollo y dinamización de los procesos de generación de conocimiento que propicie la innovación (Nonaka, et al., 2000).

\section{LA INNOVACIÓN COMO CAPACIDAD DINÁMICA PARA LAS ORGANIZACIONES}

Reinventarse en un mundo de cambios implacables, se ha convertido en una obligación para las organizaciones, donde las capacidades dinámicas permiten desarrollar nuevos productos y procesos dirigidos a responder a las necesidades del cambiante entorno (Helfat, 1997; Mendoza, 2013). 
El origen del término innovación se le atribuye al científico austriaco Schumpeter (1939), quien la describió, basándose en una función, fundamentada en los cambios de producción, en la cual los factores de desenvolvimiento son un componente clave y decisivo en el desarrollo económico de las organizaciones. Mientras, los factores materiales o productivos, pasan a un segundo plano, ya que asumen una función de apoyo para el proceso de innovación (Montoya, 2004; Consuegra \& Ariza, 2016).

La innovación es una herramienta especial, que permite a los empresarios convertir cambios inesperados en oportunidades para la organización (Drucker, 1985). Los cambios en el entorno son vistos por los innovadores como oportunidades para re-inventar productos y procesos, de acuerdo a las nuevas necesidades del mercado. Es así como los empresarios necesitan indagar sobre las fuentes de innovación, y sus orígenes, lo que indica el camino de la innovación exitosa (Van de ven, et al., 2001; Consuegra \& Ariza, 2016).

Por tanto la innovación no debe centrarse exclusivamente en los factores internos de la organización, ya que es indudable la importancia que tienen el entorno y los factores externos para una exitosa gestión (Porter \& Stern, 2001; Consuegra \& Ariza, 2016).

En ese sentido, Bower \& Christensen (1995) y Christensen (1999) se refieren a la innovación como el cambio en las tecnologías de las organizaciones donde las empresas líderes deben invertir constantemente en recursos y herramientas que les permitan mantenerse a la vanguardia, comprendiendo y adaptándose de manera rápida a lo que el entorno requiere, y visualizando las necesidades que en el futuro puedan presentarse en el medio (Christensen, 1999; Consuegra \& Ariza, 2016).

La innovación, según Roberts (1988), es entendida como el proceso de invención más comercialización. Esta postura tiene en cuenta que la idea de crear algo nuevo, deriva en el desarrollo o aplicación de un producto novedoso. Que se introduzca y sea utilizado, depende de las condiciones 
del mercado y las necesidades del entorno. Por tanto, la innovación es la posibilidad de realizar una nueva actividad, que en el pasado no se podía realizar o era limitada para un segmento de mercado, de manera más eficiente (Fernandez, 2005).

Siendo más específico, y no viendo la innovación como el simple ejercicio de comercializar, Mendoza (2006) define la combinación de creatividad con acción como el elemento perfecto para propiciarla. Estos dos factores son recíprocamente complementarios, ya que el uno depende del otro en su justa medida. Es así como una idea creativa depende en gran proporción de la manera como se pueda desarrollar o llevar a la práctica (Consuegra \& Ariza, 2016).

En ese sentido, y dando un concepto más amplio, el manual de Oslo OCDE (2006) define la innovación como la introducción de un nuevo o la mejora relevante en un producto, método de comercialización o método organizativo, proceso interno de la organización, nueva disposición o mejoramiento del lugar de trabajo o del relacionamiento con el sector externo.

La innovación, entendida como una nueva y mejor forma de llevar productos al mercado (Porter, 1990), que convierte los cambios del entorno en oportunidades para la organización (Drucker, 1985), debe desarrollarse como un proceso continuo, dirigido a potencializar sus aspectos internos, con el fin de integrar y reconfigurar recursos para desarrollar capacidades que permitan responder, adaptarse, o inclusive, crear cambios en el mercado (Eisenhardt \& Martin, 2000).

No obstante, el hecho que una organización desarrolle productos o servicios innovadores, no indica que esté desplegando capacidad dinámica de innovación. Para entender como esta se desarrolla, es relevante mencionar que las capacidades dinámicas tienen su origen en la teoría basada en recursos y capacidades (Penrose, 1959; Wernerfelt, 1984; Barney, 1991; Grant, 1991; Peteraf, 1993; Teece, et al., 1997; Rangone, 1999), quienes coinciden en que la clave para alcanzar ventaja competitiva, se basa en la habilidad de integrar los valiosos y heterogéneos recursos tangibles e intangibles disponibles en la organización. 
En ese sentido, Barney (1991) describe que para que los recursos puedan tener potencial de generar valor y crear ventaja competitiva deben cumplir las siguientes características:

- Valioso: El elemento debe tener el potencial de responder a las amenazas del entorno y aprovechar sus oportunidades, al tiempo que desarrolla fortalezas para minimizar las debilidades.

- Raro: Cumplir con la condición de particular, escaso, único o limitado entre un conjunto de competidores actuales y potenciales.

- Imperfectamente imitable: La combinación de valioso y raro, disminuye las posibilidades para que exista la imitación, al tiempo que las barreras de entrada para su obtención o adquisición son costosas o demandan demasiados recursos.

- Explotado por la organización: La organización debe contar con aspectos organizativos que permitan desarrollar el potencial de los recursos. En ese sentido, los recursos se convierten en capacidades en la medida que son adaptados, integrados y reconfigurados en procura del desarrollo de las habilidades organizacionales (Teece, et al., 1997).

Es así como las organizaciones poseen diferentes tipos de capacidades, las cuales se clasifican de acuerdo a su impacto, el valor que agregan a sus procesos y la forma de ventaja competitiva que son capaces de alcanzar (Consuegra \& Ariza, 2016). De esta manera, las capacidades dinámicas también son conocidas como de orden superior, ya que hacen referencia a las capacidades particulares, no imitables, que las organizaciones pueden desarrollar para replantear, configurar y reinventar sus recursos, con miras a estar en un constante proceso de transformación sobre los cambios tecnológicos, de modelos organizacionales y de mercado, al tiempo que se adaptan a los nuevos retos del entorno (Teece, et al., 1997).

En consecuencia, las capacidades dinámicas buscan resolver sistemáticamente los problemas de la organización basadas en dos supuestos. 
Por una parte, el de competitividad de largo plazo, resultado de poseer un conjunto de recursos con características de alto valor (valioso, raro, imperfectamente imitable y explotado por la organización) que sean imperfectamente móviles (Barney, 1991; Peteraf, 1993), es decir, que sean específicas y únicas de la organización (Consuegra \& Ariza, 2016)

Por otro lado, la adopción de rutinas organizacionales (Nelson \& Winter, 1982; Teece, et al., 1997; Eisenhardt \& Martin, 2000; Hardagon, 2002; Zollo \& Winter, 2002), con las cuales la firma puede variar, transformar y ordenar sus recursos apropiándose, sumándolos y combinándolos, para crear nuevas estrategias, dirigidas a la creación de valor (Wernerfelt, 1984; Barney, 1991; Grant, 1991; Teece, et al., 1997; Rangone, 1999; Aguilar \& Yepes, 2006), siempre permitiendo la constante adaptación, innovación y adopción de nuevos modelos para la empresa.

Por tanto, las empresas deben concebir nuevas capacidades organizacionales, a partir de la indefinida creación, ampliación, mejoramiento, protección, integración, reconfiguración, renovación, incremento y construcción de sus capacidades superiores (Wernerfelt, 1984; Prahadal \& Hamel, 1990; Grant, 1991; Barney, 1991; Nelson, 1991; Teece, et al., 1997; Rangone, 1999), las cuales buscan adaptarse a los cambios en mercados y nuevas tecnologías que incluyen la capacidad de la empresa de configurar el entorno en el que opera, creando o mejorando nuevos productos y procesos, además de diseñar e implementar modelos de negocio viables (Garzón, 2013).

Por eso, la innovación se convierte en fuente esencial para la generación de capacidades dinámicas, ya que busca que las organizaciones desarrollen nuevas soluciones que le permitan cultivar, adaptar y regenerar su conocimiento, con el fin de crear, retener y transformar sus recursos, en capacidades organizacionales de orden superior, orientando sus acciones a la creación o modificación constante de productos, procesos y métodos que buscan responder a las condiciones del entorno (Consuegra \& Ariza, 2016). 
La capacidad de innovación, es definida por Wang \& Ahmed (2007) como la habilidad de la organización para el desarrollo de nuevos productos y servicios, métodos de producción, la identificación de nuevos mercados, el descubrimiento de nuevas fuentes de suministro y el perfeccionamiento de nuevas formas organizativas (Barrios \& Olivero, 2015; 2017) mediante la alineación de las orientaciones estratégicas, comportamientos y procedimientos, los cuales deben ser innovadores (Wang \& Ahmed, 2007).

Para Lawson \& Samson (2001), la capacidad de innovación hace referencia a la habilidad para transformar continuamente el conocimiento y los planteamientos, en nuevos productos, procesos y sistemas, procurando el mayor beneficio de la organización y los Stakeholders. Es así como, las organizaciones desarrollan capacidad de innovación cuando son capaces de configurar sus recursos organizativos, principalmente los que buscan y propician el aumento y generación de conocimiento (Winter, 2003)

Acosta \& Fischer (2013) definen la capacidad de innovación como la competencia para la gestión y búsqueda intensiva de conocimiento, con el propósito de movilizar, combinar y ejecutar aquellos recursos científicos y técnicos (Nonaka \& Takeuchi, 1995; Gant, 1996; Subramaniam \& Youndt, 2005), que mediante los procesos de exploración, explotación y ambidestreza organizacional (Duncan, 1976 ; Cohen \& Levinthal, 1990; Rothaermel \& Alexandre, 2009; Teece, 2009), posibilita el desarrollo de ideas, procesos o productos con éxito (Burns \& Stalker, 1961; Thompson, 1965; Hurley \& Hult, 1998; Lawson \& Samson, 2001), para creación de estrategias competitivas, generadoras de resultados empresariales superiores en condiciones de entornos inciertos y dinámicos (Teece, et al., 1997; Helfat \& Raubistchek, 2000; Furman, et al., 2002).

En ese sentido, los procesos de la capacidad de innovación se caracterizan de acuerdo a la esencia, propiedad de los flujos de conocimiento, estrategia de la organización y las condiciones del entorno. Por una parte, está el proceso de explotación, el cual hace referencia al desarrollo de innovación incremental hasta que cambian las condiciones del mercado. 
Por otra parte, se encuentra el proceso de exploración, el cual dirige los esfuerzos de la organización en la búsqueda incesante de innovaciones radicales o disruptivas, los cuales determinan el liderazgo del mercado por un período de tiempo (Acosta \& Fischer, 2013).

Sin embargo, una organización puede desarrollar ambos procesos simultáneamente, encontrando un equilibrio entre las actividades de exploración y explotación de recursos y capacidades (Vivas, 2013), dando lugar a la ambidestreza organizacional, la cual nace como un nuevo concepto de la teoría administrativa, por la necesidad de las organizaciones de mantener un desempeño sostenido y prolongado en el tiempo (Raisch, et al., 2009).

La ambidestreza organizacional hace referencia a las rutinas y procesos mediante los cuales se movilizan, coordinan e integran conjuntamente esfuerzos de exploración y de explotación (Jansen, et al., 2009), basados en la premisa de que el éxito prolongado requerirá de estructuras duales que le permitan desarrollar su proceso de innovación, de acuerdo a las necesidades internas y externas del momento (Duncan, 1976).

Al respecto, Acosta \& Fischer (2013) creen que se desarrolla ambidestreza organizacional cuando la organización es capaz de competir en mercados maduros los cuales tienen características de costo-eficiencia, al tiempo que demuestran procesos de innovación incremental significativos y desarrollan nuevos productos y servicios para mercados emergentes, los cuales les permiten rendimientos superiores.

Sobre las bases de esas consideraciones, es evidente que la innovación es considerada como un factor clave en la competitividad de las organizaciones, por cuanto en todas las partes de la economía pueden identificarse procesos de generación de conocimiento que favorecen la creación de innovaciones (López, et al., 2011). Es así como se puede argumentar que la economía y el desarrollo de ventajas sostenibles en el tiempo de las organizaciones, van a depender en gran medida de su capacidad de innovación y la generación de nuevo conocimiento (Porter, 1990; Flórez, 2005; Bravo \& Herrera, 2009). 
Teniendo en cuenta que esta investigación, se centra en analizar la capacidad de innovación de una IES, partiendo de la premisa que estas son fuente primaria para la creación de conocimiento, es evidente que las IES tienen un papel preponderante desde su función sustantiva de extensión (mejor conocida como interacción con el entorno), en el desarrollo de dicha capacidad, a partir de la triada Universidad-Empresa-Estado, buscando elementos dinamizadores que mantengan en constante movimiento la reproducción de conocimientos compartidos entre la universidad y la sociedad (Morales, et al., 2010; Castellanos, et al., 2003).

\section{GESTIÓN DEL CONOCIMIENTO Y CAPACIDAD DE INNOVACIÓN EN IES}

Considerado como un elemento esencial de la generación y desarrollo de la capacidad de innovación, el Ba permite una mirada holística y un acercamiento a los enfoques estratégicos e integradores de los recursos y conocimiento de la organización. Es por esto que los espacios compartidos de conocimiento, deben darse como resultado de la incesante búsqueda de nuevas y buenas ideas que permitan explorar y explotar la capacidad de innovación en los procesos de la organización.

El resultado del Ba es la innovación (Kanter, 2000; Nonaka, et al., 2000; Bueno, et al., 2008), por tanto, se deben generar procesos y estrategias orientados a construir y desarrollar nuevas ideas que sean útiles para el mantenimiento y aumento de los stocks de conocimiento y los flujos de aprendizaje de la organización (Acosta, et al., 2014). Las organizaciones deben en primera instancia conocerse desde adentro, ahondando en la comprensión de sus recursos estratégicos, con el fin de poder tener las bases para explorar y explotar sus recursos, de acuerdo a las condiciones del entorno dinámico.

De acuerdo a esto, el Ba se concibe como el espacio donde interactúan de manera dinámica el conocimiento tácito y el conocimiento explícito, y todas sus modalidades de combinación. Desde esta perspectiva, el Ba puede considerarse como un nivel de conocimiento individual y colectivo en 
desarrollo, que se ocasiona con la interacción de las personas de un equipo de trabajo y su entorno (Acosta, 2009).

Para el Ba no existen fronteras, ya que este se puede presentar a través de las redes organizacionales que facilitan el intercambio y creación del conocimiento. Estas redes pueden ser físicas, virtuales, mentales, o su combinación (Nonaka \& Konno, 1998). De la fuerza y consolidación que tengan estas redes, dependerán los niveles de confianza, compromiso y creatividad que exista entre los miembros que las componen (Acosta, et al., 2014; Acosta, 2010).

En ese sentido, las IES son organizaciones adecuadas para impulsar los distintos tipos de $\mathrm{Ba}$, porque permiten la generación y difusión de nuevo conocimiento en todos los niveles. De igual manera, estas deben contar con una estructura flexible y la apropiación de una cultura innovadora que promueva la continua generación, análisis, transformación y propagación de conocimiento tácito (García, 2016). Por tanto, generar conocimiento novedoso requiere la alineación del diseño de la estructura organizativa, el Ba y la estrategia organizacional (Nonaka \& Konno, 1998; Von Krogh, et al., 2012; Nonaka, et al., 2014).

Una de las características principales del Ba es su dinamismo, ya que puede aparecer y desaparecer en cualquier momento. De igual manera, su flexibilidad, ya que no existe un espacio determinado y fijo para su generación. Su potencial obedecerá a la constancia y disposición de los miembros de la organización en las interacciones que se den a nivel de individuos y grupos, del tipo y calidad de conocimiento que se intercambie colectivamente y de la comunicación que emerja entre las partes (Von Krogh, et al., 2000; Acosta, et al., 2014; Nonaka, et al., 2014).

El Ba implica tener en cuenta una serie de consideraciones con respecto al contexto, los propósitos de la organización, los factores organizacionales, la cultura organizacional, la forma de liderazgo, las acciones e interacciones que se generen entre los diferentes actores, la infraestructura, entre otros. Estos elementos contribuyen a la acción porque implican mover recursos 
y generar nuevo conocimiento a partir de información, valores, ideas, experiencias, emociones y creencias que se comparten de manera voluntaria (Fayard, 2005), y con cuya interacción se pretende responder a las necesidades del entorno.

De esta manera, la interacción entre la IES y el entorno, ha tomado una fuerte relevancia en los últimos años, derivado de la importancia que estas tienen en la actual sociedad del conocimiento (Etzkowitz \& Leydesdorff, 2000; Casallas, et al., 2011), donde se les reconoce como agente integrador y fuente esencial para el entendimiento sistémico de la realidad social (Clark, 1983).

Es así como los países desarrollados han entendido la importancia de la consecución y perfeccionamiento de alianzas estratégicas con las IES, el apoyo económico por parte del Estado y la generación de una normativa acorde al contexto (CINDA, 2015; Pineda, et al., 2011), las cuales son fundamentales para el impulso de la capacidad de innovación.

Sin embargo, esta premisa es aún muy lejana en algunos países de América Latina, donde los aspectos culturales, el poco apoyo del Estado, la normatividad vigente, la desarticulación entre actores (Universidad-Empresa-Estado), la disparidad en la concepción para dar respuesta a las necesidades sociales de las regiones y la poca adaptabilidad a los cambios del entorno (Morales, et al., 2012; Cabrera, et al., 2014) han limitado el desarrollo de la capacidad de innovación en las IES.

En ese orden de ideas, las investigaciones (Villaveces, 2006; Ramírez \& García, 2010; Barrios \& Olivero, 2015; Lundvall, 2010; Etzkowitz \& Leydesdorff, 1995; Clark, 1983), han demostrado que para el desarrollo de la innovación como capacidad se requiere de la construcción de entornos colaborativos, que se generen a partir de las relaciones entre el exterior y el interior de la IES, la relación entre las subunidades dentro de la IES, y la relación entre los individuos dentro de cada subunidad de la IES (Cohen \& Levinthal, 1990; Barrios \& Olivero, 2015).

En definitiva, el desafío de las IES consiste en promover entre los dife- 
rentes miembros de la organización, espacios de conocimiento que generen resultados favorables no solo al interior de la institución, sino que impacten de manera positiva el entorno en el que desarrollan sus actividades (Clark 1983).

Por tanto, la implementación para la exploración y explotación de la capacidad de innovación en la organización, depende de qué tan preparados están los miembros para los cambios del entorno y cómo se desarrolla la cultura de la IES (Sorensen, 2002), ya que esta determina qué tan preparados se encuentran para enfrentarse a la innovación.

Una cultura innovadora fuerte implica que los miembros de la organización compartan una intensa dedicación de los valores, rutinas e ideas sobre innovación, encaminados a la búsqueda incesante del propósito estratégico. Por ello, se hace necesario poder cambiar las habilidades y prácticas orientadas a construir una cultura innovadora, acorde a las singularidades de la organización (BBVA, 2011).

De igual manera, la cultura innovadora es muy importante para los espacios compartidos de conocimiento (Ba), ya que busca el aprovechamiento eficiente del conocimiento organizativo y el desarrollo de capacidades al interior de la organización. Esto hace que bien aprovechados, los comportamientos que se derivan de la cultura innovadora garanticen la disponibilidad y movilidad de flujos y stocks de conocimiento en general, así como la creación y desarrollo de la capacidad de innovación (Acosta, 2010). De esa forma, la generación del espacio compartido de conocimiento (Ba), se verá favorecido por una cultura y un ambiente de trabajo que estimule la comunicación y los flujos de información dentro de la empresa (Amabile, 1997).

En el caso de las IES, la cultura es la que permitirá la adaptación a los cambios requeridos para desarrollar la capacidad de innovación. Por ello, la cultura innovadora debe propender por flexibilizar sus funciones sustantivas, permitiéndole actuar de manera efectiva para cumplir con los compromisos de la filosofía institucional (Clark, 1998). De esta manera, la cultura innovadora en las IES debe ser un proceso en permanente evolución, 
construcción y transformación, el cual sirva como herramienta efectiva para el crecimiento y productividad de la Institución (Sporn, 2001).

Sin embargo, uno de los retos de la capacidad de innovación y la gestión del conocimiento, radica en hacer compatibles los cambios que esta representa, teniendo en cuenta la tradición, influencia y prestigio de las IES (cuando algunas innovaciones pueden parecer suponer un peligro para las particularidades e identidad de las instituciones), implementando estrategias originales que reconozcan lo que es esencial y específico a cada una de ellas para basarse en ese capital (Dondi, 2008) y explotarlo de manera innovadora.

\section{METOdOLOGÍA}

La investigación se llevó a cabo en una institución de educación superior de la ciudad de Barranquilla, durante los meses comprendidos entre abril y octubre de 2016.

La investigación desarrollada fue de tipo descriptiva y exploratoria, puesto que permitió aplicar un conjunto de métodos y procedimientos científicos para recolectar datos puros, y generar estructuras que permitieron analizar la gestión del conocimiento y capacidad de innovación de la Universidad a partir de la interacción con el entorno.

Además, el carácter de investigación exploratoria se debe a que existen pocas evidencias de estudios al respecto y este trabajo servirá para la formulación de investigaciones con mayor nivel de profundidad.

Para el caso de esta investigación, la población estuvo conformada 140 miembros de la Universidad y del sector externo. Teniendo en cuenta que en la última década las IES están afrontando cambios significativos en el entorno y la evidencia científica es escasa, se decidió centrar la investigación en una sola Institución.

Esta IES en particular, se encuentra fortaleciendo su política de relación 
con el sector externo, mediante el desarrollo de rutinas innovadoras y la ejecución de proyectos en conjunto, que respondan a los cambios previstos en el entorno, lo que indica que requieren apoyo para el desarrollo de la gestión del conocimiento y capacidad de innovación

Para la recolección de datos, se elaboró un listado fiable de los miembros de la Universidad. La relación fue tomada de la nómina entregada por el Departamento de Recursos Humanos y Servicios Externos, de la cual se seleccionaron 140 personas, entre quienes ocupan cargos de dirección, pertenecen a la Vicerrectoría de Investigación y miembros del sector productivo (Véase Tabla 1).

Tabla 1. Ficha de investigación

\begin{tabular}{|l|l|}
\hline UNIVERSO DE LA INVESTIGACIÓN & Una Institución de Educación Superior \\
\hline ÁMBITO GEOGRÁFICO & Barranquilla, departamento del Atlántico \\
\hline POBLACIÓN & $\begin{array}{l}140 \text { personas entre directivos, académicos, investiga- } \\
\text { dores y miembro del sector productivo }\end{array}$ \\
\hline MUESTRA & $100 \%$ de la población \\
\hline $\begin{array}{l}\text { TÉCNICA DE RECOLECCIÓN DE LA } \\
\text { INFORMACIÓN }\end{array}$ & Encuesta \\
\hline PROCESAMIENTO DE MUESTREO & Cuestionario \\
\hline NÚMERO DE CUESTIONARIOS & 140 \\
\hline TASA DE RESPUESTA & $100 \%$ \\
\hline PERÍODO & abril, 2016 - Octubre, 2016 \\
\hline \multicolumn{2}{|c|}{ Fuente: Elaboración propia }
\end{tabular}

Por otra parte, se consideró la encuesta como técnica apropiada para la recogida de datos. Esta se considera una herramienta efectiva y válida para reunir información sobre un tema donde hay escasas evidencias. El objetivo de la técnica es analizar las opiniones de un grupo con características similares, a través de un cuestionario que responde a unos ítems previamente elaborados y validados por un grupo de tres expertos.

El instrumento elegido para la recopilación de la información ha sido un cuestionario. El cuestionario se compone de 15 ítems. Para ello, han sido 
determinantes aspectos como la claridad y brevedad. Previo a la presentación del cuestionario final, se realizó la operacionalización de las variables en el cuadro metodológico, donde se establecieron las variables a medir por la investigación, con sus respectivos indicadores.

En lo referente a la medición del concepto capacidad de innovación, la redacción de las variables se ha basado en los resultados obtenidos de la revisión del marco teórico. De esta forma, se ha pretendido analizar cómo se está gestionando el conocimiento para el desarrollo de la capacidad de innovación en la Universidad, a partir de la interacción con el entorno.

Se utilizó una escala tipo Likert en el cuestionario (siempre, casi siempre, algunas veces, casi nunca y nunca). Este tipo de escala permite medir la reacción del sujeto ante el ítem, el cual se presenta como la propiedad que el investigador desea medir (Padua, 1979; Garzón, 2013).

Posteriormente, se determinó la validez y la confiabilidad. La validez se refiere al grado en que un instrumento realmente mide la variable que pretende medir. El análisis efectuado por los expertos incluyó la evaluación de la pertinencia o no pertinencia de los objetivos específicos y su relación con las variables y los ítems. Además, evaluaron la redacción del instrumento elaborado. Por su parte, en el análisis de confiabilidad se obtuvo un Alpha de 0.942 con 15 ítem válidos, generados por el procedimiento análisis de fiabilidad de la herramienta SPSS, por lo que se consideró que la escala es fiable.

\section{RESULTADOS}

Con el fin de verificar los resultados de los datos en cuanto a forma, se realizó un análisis de simetría, curtosis y desviación estándar, con lo cual se busca darle mayor precisión sobre el instrumento aplicado (Véase Tabla 1). 
Tabla 1. Variables de la investigación

\begin{tabular}{|c|c|c|c|}
\hline VARIABLE & SUBVARIABLE & AFIRMACIÓN & $\begin{array}{l}N^{\circ} \mathrm{DE} \\
\text { ITEM }\end{array}$ \\
\hline \multirow{6}{*}{$\begin{array}{l}\text { Capacidad de } \\
\text { innovación }\end{array}$} & \multirow{3}{*}{ Exploración } & $\begin{array}{l}\text { La Universidad utiliza métodos o instrumentos de vigilan- } \\
\text { cia del entorno con el fin de adaptarse rápidamente a las } \\
\text { condiciones cambiantes. }\end{array}$ & 1 \\
\hline & & $\begin{array}{l}\text { La Universidad utiliza métodos o instrumentos de vigilancia } \\
\text { del entorno con el fin de adaptarse rápidamente a las condi- } \\
\text { ciones cambiantes. }\end{array}$ & 2 \\
\hline & & $\begin{array}{l}\text { La Universidad mantiene contacto permanente con gremios y } \\
\text { asociaciones profesionales para desarrollar proyectos innova- } \\
\text { dores de forma conjunta o asociativa. }\end{array}$ & 3 \\
\hline & \multirow{3}{*}{ Explotación } & $\begin{array}{l}\text { La Universidad dispone de personas con responsabilidad y } \\
\text { asignación de recursos para la prospección y comprensión del } \\
\text { entorno. }\end{array}$ & 4 \\
\hline & & $\begin{array}{l}\text { La Universidad dispone de personas con responsabilidad y } \\
\text { asignación de recursos para la realización de estudios de desa- } \\
\text { rrollo de negocios productivos (creación y mejora de produc- } \\
\text { tos, servicios, mercados, etc.). }\end{array}$ & 5 \\
\hline & & $\begin{array}{l}\text { El conocimiento en la Universidad se utiliza para desarrollar } \\
\text { productos y servicios nuevos y mejorados. }\end{array}$ & 6 \\
\hline \multirow{10}{*}{$\begin{array}{c}\text { Gestión del } \\
\text { Conocimiento }\end{array}$} & \multirow{10}{*}{$\mathrm{Ba}$} & $\begin{array}{l}\text { En la Universidad se fomenta la creación de equipos de trabajo } \\
\text { interdisciplinarios de diferentes dependencias. }\end{array}$ & 7 \\
\hline & & $\begin{array}{l}\text { La Universidad proporciona los medios necesarios para com- } \\
\text { partir conocimiento entre las diferentes dependencias. }\end{array}$ & 8 \\
\hline & & $\begin{array}{l}\text { En la Universidad se valora y se fomenta la aportación de nue- } \\
\text { vas ideas. }\end{array}$ & 9 \\
\hline & & $\begin{array}{l}\text { El nivel de formación de los colaboradores de la Universidad, } \\
\text { permite asimilar fácilmente nuevos conocimientos y asumir } \\
\text { los cambios del entorno. }\end{array}$ & 10 \\
\hline & & $\begin{array}{l}\text { Las directivas de la Universidad promueven el desarrollo de la } \\
\text { capacidad de innovación. }\end{array}$ & 11 \\
\hline & & $\begin{array}{l}\text { Las soluciones y recomendaciones propuestas por los colabo- } \\
\text { radores son utilizadas para desarrollar nuevas prácticas, pro- } \\
\text { cesos, productos, servicios, etc. }\end{array}$ & 12 \\
\hline & & $\begin{array}{l}\text { La Dirección de Talento Humano promueve el desarrollo inte- } \\
\text { gral de los colaboradores. }\end{array}$ & 13 \\
\hline & & $\begin{array}{l}\text { En la Universidad existe un compromiso permanente con la } \\
\text { innovación. }\end{array}$ & 14 \\
\hline & & $\begin{array}{l}\text { Los directivos apoyan el desarrollo de proyectos innovadores, } \\
\text { aunque impliquen cierto grado de riesgo. }\end{array}$ & 15 \\
\hline & & $\begin{array}{l}\text { En la Universidad se promueve y estimula la confianza de los } \\
\text { colaboradores. }\end{array}$ & 16 \\
\hline
\end{tabular}

Fuente: Elaboración propia 
Respecto a la variable espacio compartido de conocimiento, se deja en evidencia que, tanto la curtosis y la asimetría se encuentran lo más cercano a cero, con una media próxima a 4, y una desviación estándar mayor a 0,75. Por tanto, los datos de las variables del factor espacio compartido de conocimiento están distribuidos normalmente en términos de medidas de forma (asimetría y curtosis) (Véase Tabla 2).

Tabla 2. Espacio Compartido de Conocimiento

\begin{tabular}{|l|c|c|c|l|c|l|l|}
\hline & C & MEDIA & M.ABSOL & D.ESTÁNDAR & $\begin{array}{c}\text { R.INTER- } \\
\text { CUARTIL }\end{array}$ & ASIMETRÍA & CURTOSIS \\
\hline Ítem 6 & ECC & 4,092857 & 4 & 0,9810905 & 1 & $-0,8840326$ & $-0,029097706$ \\
\hline Ítem 7 & ECC & 3,721429 & 4 & 0,9968862 & 1,25 & $-0,4312085$ & $-0,277391111$ \\
\hline Ítem 8 & ECC & 3,964286 & 4 & 0,8929034 & 2 & $-0,4830867$ & $-0,567455639$ \\
\hline Ítem 9 & ECC & 4,092857 & 4 & 0,7576604 & 1 & $-0,4587863$ & $-0,261727085$ \\
\hline Ítem 10 & ECC & 4,128571 & 4 & 0,9043688 & 1 & $-0,8500441$ & 0,224210012 \\
\hline Ítem 11 & ECC & 3,870504 & 4 & 0,8060867 & 1 & $-0,6016573$ & 0,603716791 \\
\hline Ítem 12 & ECC & 3,771429 & 4 & 0,9471111 & 1 & $-0,6600224$ & 0,50293269 \\
\hline Ítem 13 & ECC & 4,228571 & 4 & 0,7987657 & 1 & $-0,7802827$ & $-0,009522542$ \\
\hline ítem 14 & ECC & 3,871429 & 4 & 0,8121634 & 1 & $-0,5768113$ & 0,509566582 \\
\hline Ítem 15 & ECC & 4,021429 & 4 & 0,7998908 & 1 & $-0,5521569$ & $-0,055281362$ \\
\hline
\end{tabular}

Fuente: Elaboración propia

Lo anterior indica que la población a la cual se le aplicó el instrumento considera que en la Universidad se fomenta la creación de equipos de trabajo interdisciplinarios de diferentes dependencias y que en la Institución se valora y se fomenta la aportación de nuevas ideas, las cuales son utilizadas para desarrollar nuevas prácticas, procesos, productos, servicios, etc. Asimismo, la IES proporciona los medios necesarios para compartir conocimiento entre las diferentes dependencias; de esta manera, se promueve el desarrollo de la capacidad de innovación. Por otra parte, el nivel de formación de los colaboradores de la Universidad, permite asimilar fácilmente nuevos conocimientos y asumir los cambios del entorno.

Seguidamente, el análisis de la capacidad de innovación permitió evidenciar de igual forma que la curtosis y la asimetría se encuentran lo más 
cercano a cero, con una media próxima a 4 y una desviación estándar mayor a 0,78. Por tanto, los datos de la variable están distribuidos normalmente en términos de medidas de forma (Véase Tabla 3).

Tabla 3. Exploración y Explotación

\begin{tabular}{|l|c|c|l|c|l|l|}
\hline & MEDIA & M.ABSOL & D.ESTÁNDAR & R.INTERCUARTIL & ASIMETRÍA & CURTOSIS \\
\hline Ítem 1 & 3,642857 & 4 & 0,8655803 & 1 & $-0,3155751$ & $-0,175955376$ \\
\hline Ítem 2 & 4,307143 & 4 & 0,7856302 & 1 & $-0,7843104$ & $-0,36031268$ \\
\hline Ítem 3 & 3,842857 & 4 & 10.055 .345 & 2 & $-0,6259847$ & $-0,271716129$ \\
\hline Ítem 4 & 3,978571 & 4 & 0,9013807 & 2 & $-0,4956805$ & $-0,603171074$ \\
\hline Ítem 5 & 3,828571 & 4 & 0,864511 & 1 & $-0,2018332$ & $-0,735229146$ \\
\hline
\end{tabular}

Fuente: Elaboración propia

En consecuencia, la investigación demostró que la capacidad de innovación se caracteriza por desarrollar simultáneamente procesos de exploración y explotación mediante al ambidestreza organizacional. Es así como la IES es capaz de utilizar métodos o instrumentos de vigilancia del entorno con el fin de adaptarse rápidamente a las condiciones cambiantes; además, mantiene contacto permanente con gremios y asociaciones profesionales para desarrollar proyectos innovadores de forma conjunta o asociativa.

Por otro lado, los resultados demuestran que la IES dispone de personas con responsabilidad y asignación de recursos para la prospección y comprensión del entorno y de personas con responsabilidad y asignación de recursos para la realización de estudios de desarrollo de negocios productivos (creación y mejora de productos, servicios, mercados, etc.). Por eso, se deduce que el conocimiento en la Universidad se utiliza para desarrollar productos y servicios nuevos y mejorados.

\section{CONSIDERACIONES FINALES}

En primera instancia, se encuentra que a pesar que la institución ha tenido grandes avances con respecto a la explotación de su capacidad de innovación, este esfuerzo muchas veces es resultado de la voluntad aislada 
de sus miembros, los cuales desean mostrar el camino de las universidades emprendedoras (Clark, 1998; 2004).

En este orden de ideas, es evidente el fortalecimiento de un propósito estratégico, en el cual se incluye la innovación como eje central del plan estratégico, al igual, existe un desconocimiento por parte de algunos miembros de la organización sobre las ventajas de la innovación para las IES. Prueba de ello, es que algunos de estos miembros a los que se les fue enviado el formulario, tienen ciertos arraigos sobre la investigación social y científica tradicional, con conceptos erróneos sobre lo que es la innovación y su importancia para el desarrollo de capacidades superiores en la Universidad.

En ese sentido, esto denota la necesidad de fomentar una cultura innovadora que sea vivida por los miembros de la organización y que permita que la innovación haga parte del ADN institucional. Sin embargo, se evidenció la existencia de rasgos culturales, los cuales han sido asumidos con el tiempo por los miembros, como parte de rutinas organizacionales.

Cabe resaltar que este rasgo de cultura no es exclusivo de la Universidad en estudio, como bien lo mencionan Morales, et al. (2012). Colombia y los países de América Latina, demuestran que los aspectos culturales y normativos, la desarticulación de la tríada Universidad-Empresa-Estado, las discrepancias en la forma de responder a las necesidades sociales de cada región y la carencia de una política sobre la propiedad intelectual, limitan y dificultan la creación de condiciones propicias para la generación de innovaciones.

Es importante resaltar que la Universidad como organización generadora de conocimiento, es un lugar favorable para el desarrollo de ventajas competitivas a través de la innovación, especialmente alrededor de su función sustantiva de extensión, la cual es la responsable de articular los desarrollos que son realizados por docentes e investigadores con el entorno.

Sobre la interacción de la Universidad con el sector externo, la cual es manejada de diferentes maneras con resultados positivos en los últimos 
años, se sugiere encontrar un método efectivo para involucrar a los profesores investigadores con las realidades del entorno y requerimientos específicos de los sectores productivos de la sociedad, para de esta forma, hallar, por medio de sus proyectos de investigación, soluciones prácticas e innovadoras para el entorno (Ramírez \& García, 2010).

Por último, es importante resaltar la decisión de la alta dirección para el apoyo del emprendimiento, el cual ha sido concebido como una estrategia para fomentar la cultura innovadora en todos los miembros de la organización, creando una propuesta innovadora, que engendra valor y propende por el encuentro de la Universidad, la Empresa y el Estado, generando resultados favorables y de alto impacto, los cuales esperamos se sigan fortaleciendo con el pasar de los años.

\section{REFERENCIAS BIBLIOGRÁFICAS}

Acosta, J. (2009). Espacios de conocimiento. Contexto para el desarrollo de capacidades tecnológicas. Boletín Intellectus, (15), 12-18.

Acosta, J. (2010). Creación y desarrollo de capacidades tecnológicas: Un modelo de análisis basado en el enfoque de conocimiento. Madrid: Universidad Autónoma de Madrid.

Acosta, J. \& Fischer, A. (2013). Condiciones de la gestión del conocimiento, capacidad de innovación y resultados empresariales. Un modelo explicativo. Pensamiento y gestión, 35, 25-63.

Acosta, J., Zárate, R., \& Luiz, A. (2014). Ba: espacios de conocimiento. Contexto para el desarrollo de capacidad de innovación. Un análisis desde la gestión del conocmiento. Revista EAN, (76), 44-63.

Aguilar, J., \& Yepes, E. (2006). Gestión de capacidades dinámicas e innovación: Una aproximación conceptual. Revista de ciencias da administracao, (8), 1-15.

Amabile, T. (1997). Motivating Creativity in Organizations: On Doing What You Love and Loving What You Do. California Management Review, 40 (1), 39-58. 
Barney, J. (1991). Firm resources and sustained advantage. Journal of management, (17), 99-120.

Barrios-Hernández, K., \& Olivero-Vega, E. (2015). La innovación en instituciones de educación superior: Un modelo basado en capacidades dinámicas. Barranquilla, Colombia: Universidad Simón Bolívar.

Barrios-Hernández, K., Olivero-Vega, E. \& Acosta-Prado, J. (2017). Capacidad dinámica de innovación en instituciones de educación superior. Revista Espacios, 38(01), 1-24.

Bower, J. \& Christensen, C. (1995). Disruptive Technologies: Catching the Wave. Harvard Business Review, 73, 43-53.

Bravo, E. \& Herrera, L. (2009). Capacidad de innovación y configuración de recursos organizativos. Intangible capital, 5(3), 301-320.

Bueno, E. \& Morcillo, P. (1997). Dirección estratégica por competencias básicas distintivas: Propuesta de un modelo. Madrid: Instituto Universitario de Administración de Empresas.

Bueno, E., Morcillo, P. \& Salmador, M. (2006). Distinctions that matter: a classification of resources and discussion of implications for dynamic capabilities of firms. International Journal Technology Management, 41, (1-2), 155-168.

Bueno, E., Rodríguez, J. \& Salmador, M. (2008). Knowledge creation as a dynamic capability: implications for innovation management and organisational design. International Journal Management Practice, 2(1), 72-82.

Burns, T. \& Stalker, G. (1961). The management of innovation. Londres: Tavistock.

Cabrera, M., Nieto, L. \& Giraldo, R. (2014). La universidad colombiana y la innovación desde una Epistemología del Sur. Entramado, 1(10), 240-250.

Casallas, C., Plata, P. \& Pineda, K. (2011). Parques Tecnológicos como Mecanismo de Integración entre Universidades, Empresas y el Estado: retos para Colombia. Memoria VI Congreso Internacional de la Red de 
Investigación y Docencia en Innovación Tecnológica-RIDIT, Manizales, Colombia.

Castellanos, O., Chávez, R. \& Jiménez, C. (2003). Propuesta de formación en liderazgo y emprendimiento. Innovar, Revista de Ciencias Administrativas y sociales, 22, 145-156.

Christensen, C. M. (1999). El dilema de los innovadores. Buenos Aires: Ediciones Granica S.A.

CINDA, C. I. (2015). La transferencia de I+D, la innovación y el emprendimiento en las universidades. Educación superior en Iberoamérica - Informe 2015. Santiago de Chile: RIL ${ }^{\circledR}$ editores.

Clark, B. (1983). El sistema de educación superior. Una visión comparativa de la organización académica. México: Universidad Autónoma Metropolitana.

Clark, B. (1998). Creating entrepreneurial universities: Organizational pathways of transformation (No. 378 CLA).

Clark, B. (2004). Sustaining Change in Universities, Society for Research into Higher Education. Londres: Open University Press.

Cohen, W. \& Lenvinthal, D. (1990). Absorptive capacity: A new perspective on learning and innovation. Administrative science quarterly, 35, 128-152.

Consuegra, I., \& Ariza, C. (2016). La capcidad de innovación de la Universidad Simón Bolívar, a partir de la interacción con el sector externo. Barranquilla, Colombia: Universidad Simón Bolívar.

Duncan, R. (1976). the ambidextrous organization: Designing dual structures for innovation. En R. H. Kilmann, L. R. Pondy, and D. Slevin (Eds.), The management of organizaion design: Strategies and implementation (pp.167-188). New York: North Holland.

Dondi, C. (2008). Políticas europeas de apoyo a la "Open and Distance Learning" (ODL) y el caso específico de la integración de ODL en los entornos universitarios convencionales. Cuadernos IRC, (8) with the coordination by Universitat Oberta de Catalunya. 
Drucker, P. (1985). Innovation and entrepreneurship. Reino Unido: Butterworth-Heinemann.

Eisenhardt, K., \& Martin, J. (2000). Dynamic capabilities: What are they? Strategic Management Journal, 10-11(21), 1105-1121.

Etzkowitz, H., \& Leydesdorff, L. (2000). The Dynamics of Innovation: from National Systems and "Mode 2" to a Triple Helix of University-Industry-Government Relations. Elsevier Science, 29(2), 109-123.

Fayard, P. (2005). Comunidades estratégicas de conocimiento. El concepto ba en la vía japonesa hacia la creación de conocimiento. Revista Telos: Cuadernos de comunicación, tecnología y sociedad, (62).

Fernández, E. (2005). Estrategia de innovación. Madrid: Thomson Editores Spain Paraninfo SA.

Flórez, M. (2005). Gerencia del conocimiento: Su relación con la generación de capacidades innovativas. Revista de Ciencias Sociales, 11(2), 229-245.

Furman, J., Porter, M., \& Stern, S. (2002). The determinants of national innovative capacity. Research Policy, 31(2002), 899-933.

García, A. (2016). Creación, conversión, facilitación y espacios del conocimiento: las aportaciones de Ikujiro Nonaka a la teoría organizacional. Entreciencias, 4(9), 73-88.

Garzón, M. (2013). El constructo capacidades dinámicas. Ide@s CONCYTEG, 8(99), 851-870.

Grant, R. (1991). The resource-based theory of competitive advantage: implications for strategy formulation. California Management Review, 33(3), 114-135.

Grant, R. (1996). Prospering in dynamically-competitive environments: Organizational capability as knoeledge integration. Organization Science, 7(4), 375-387.

Hardagon, A. (2002). Brokering Knowledge: Linking Learning and Innovation on. Research in organizational behavior, (24), 41-85.

Helfat, C. (1997). Know-how and asset complementarity and dynamic ca- 
pability accumulation: The case of R\&D. Strategic Managment Journal, 18(5), 339-360.

Hurley, R., \& Hult, G. (1998). Innovation, market orientation, and organizational learning; an integration and empirical examination. Journal of marketing, 62(3), 42-54.

Helfat, C., \& Raubitschek, R. (2000). Product sequencing: Co-evolution ok knowledge, capabilities and products. Strategic Management Journal, 21, 961-979.

Jansen, J., Tempelaar, M., Van den Bosch, F., \& Volberda, H. (2009). Structural Differentiation and Ambidexterity: The Mediating Role of Integration Mechanisms. Organization Science, 20(4), 797-811.

Kanter, R. (2000). When a Thousand Flowers Bloom: Structural, Collective, and Social Conditions for Innovation in Organization. Research in Organizational Behavior, (22).

Lawson, B., \& Samson, D. (2001). Developing innovation capability in organizations: A dynamic capabilities approach. International Journal of Innovation Management, 5(3), 377-400.

López, M., Serrano, A., \& García, P. (2011). Análisis de la capacidad de innovación regional: el caso español. Revista Venezolana de Gerencia, 209-232.

Lundvall, B. (2010). National Systems of Innovation: Toward a Theory of Innovation and Interactive Learning. New York: Bengt-Ake Lundvall.

Mendoza, J. (2006). Innovación por lo alto. Imaginación y acción en la empresa. Bogotá: Asesores del 2000.

Mendoza, J. (2013). La capacidad dinámica de ripostar en la empresa: Confrontar entornos volátiles. Cuadernos de administración, 26(47), 63-85.

Misra, D. C. (2007). Ten Guiding Principles for Knowledge Management in E-government in Developing Countries. First International Conference on Knowledge Management for Productivity and Competitiveness. New Delhi. 
Montoya, O. (2004). Shumpeter, innovación y determinismo tecnológico. Scientia et technica, año X, 25, 209-213.

Morales, M., Mira, G., \& Arias, M. (2010). Enfoques y restos de la función de extensión universitaria, como mecanismo de integración: Universidad, empresa, Estado. II Congreso Internacional de Gestión Tecnológica e Innovación, Bogotá.

Morales, M., Pineda, K. \& Avila, K. (2012). Organizaciones innovadoras a partir de la interacción con la universidad: casos exitosos. Estudios gerenciales, (28), 363-374.

Na Ubon, A. \& Kimble, C. (2002). Knowledge Management in Online Distance Education, in Proceedings of the 3rd International Conference Networked Learning 2002, University of Sheffield, UK.

Nelson, R., \& Winter, S. (1982). An evolutionary theory of economic change. Cambridge, Massachusetts, London: Harvard University Press.

Nishida, K. (1921). An Inquiry into the Good. (M. a. Abe, Trad.) New Haven: Yale University Press.

Nonaka, I., \& Konno, N. (1998). The concept of "BA": Building a foundation for knowledge creation. California management review, 40(3), 40-54.

Nonaka, I., Toyama, R., \& Konno, N. (2000). SECl, Ba and Leadership: a Unified Model of Dynamic Knowledge Creation. Long Range Planning, 33, 5-34.

Nonaka, I., \& Takeuchi, H. (1995). The Knowledge-creating company. Oxford: Oxford University press.

Nonaka, I., Mitsuru, K., Ayano, H., \& Kohlbacher, F. (2014). Dynamic fractal organizations for promoting knowledge based transformation: a new paradigm for organizational theory. European Management Journal, 32, 137-146.

OCDE (2006). Manual de Oslo: Guía para la recogida e interpretación de datos. Madrid: Empresa de transformación agraria S.A.

Padua, J. (1979). Técnicas de Investigación aplicadas a las Ciencias Sociales. México D.F: Trillas. 
Penrose, E. (1959). The theory of the growth of the firm. Oxford: Basil Blackwell.

Peteraf, M. (1993). The cornerstones of competitive advantage: A resource bases view. Strategic Management Journal, 14, 179-191.

Pineda, K., Morales, M., \& Ortiz, M. (2011). Modelos y mecanismos de interacción universidad-empresa-estado: retos para las universidades colombianas. Equidad Desarrollo, 15, 41-67.

Porter, M. (1990). The Competitive Advantage of Nations. New York: Free Press.

Porter, M., \& Stern, S. (2001). Innovation: Location Matters. Summer. MIT Sloan Management Review, 42(4), 28-36.

Prahadal, G., \& Hamel, G. (1990). The core competence of the corporation. Harvard business review, 68(3), 79-91.

Ramírez, M., \& García, M. (2010). La alianza-Empresa-Estado: una estrategia para promover innovación. Revista EAN, 68, 112-133.

Rangone, A. (1999). Resoure-based approach to strategy analysis in smallmedium sized enterpreises. Small business economics, (12), 233-248.

Raisch, S., Birkinshaw, J., Probst, G., \& Tushman, M. L. (2009). Organizational Ambidexterity: Balancing Explotation and Exploration for Sustained Performance. Organization Science, 20(4), 685-695.

Rothaermel, F., \& Alexandre, M. (2009). Ambidexterity in technology sourcing. Organization Science, 20(4), 759-780.

Roberts, E. (1988). What we've leraned:managing invention and innovation. Research Management, 1(31), 11-29.

Sábato, J., \& Botana, N. (1968). Science and Technology in the Future Development of Latin America. Bellagio (Italy): Paper presented to The World Order Models Conference.

Shimizu, H. (1995). Ba-Principle: New Logic for the Real-time Emergence of Information. Holonics, 5(1), 67-69.

Schumpeter, J. (1939). Cycles Business. New York: McGraw-Hill. 
Sporn, B. (2001). Building adaptive universities: Emerging organisational forms based on experiences of European and US universities. Tertiary Education and Management, 7(2), 121-134.

Sorensen, J. (2002). The strength of corporate culture and the reliability of firms performance. Administrative scince quarterly, 47, 70-91.

Subramanian, M., \& Youndt, M. (2005). The influence of intellectual capital on the types of innovative capabilities. Academy of management journal, 48, 450-463.

Teece, D. (2009). Dynamic capabilities \& strategic management. Organizing for innovation and growth. Oxford: Oxford University Press.

Teece, D. J., Pisano, G., \& Shuen, A. (1997). Dynamic capabilities and strategic management. Strategic management journal, 18(7), 509-533.

Thompson, V. A. (1965). Bureaucracy and innovation. Administrative Science Quarterly, 5, 1-20.

Van de ven, A. H., Polley, D., Raghy, G., \& Venkataraman, S. (2001). El viaje de la innovación: El desarrollo de una cultura organizacional para innovar. Ciudad de México: Oxford University Press México.

Villaveces, J. (2006). Nuevas políticas de ciencia y tecnología. En H. Vessuri, Universidad e investigación científica (pp.193-205). Buenos Aires.

Vivas, S. (2013). Implicaciones de las capacidades dinámicas para la competitividad y la innovación en el siglo XXI. Cuadernos de administración, 26(47), 119-139.

Vila, J. (2011). Cultura innovadora: valores, principios y prácticas de primeros ejecutivos en empresas altamente innovadoras. En innovación y perspectivas para el siglo XXI (pp.255-267). Madrid. BBVA

Von Krogh, G., Nonaka, I., \& Reschsteiner, L. (2012). Leadership in Organizational Knowledge Creation: A Review and Framework. Journal of Management Studies, 49(1), 240-277.

Wang, C., \& Ahmed, P. (2007). Dynamic capabilities: A review and research agenda. International Journal of Management Reviews, 9(1), 31-51. 
GESTIÓN DEL CONOCIMIENTO Y CAPACIDAD DE INNOVACIÓN

Modelos, Sistemas y Aplicaciones

Wernerfelt, B. (1984). Resource-based view of the firm. Strategic Management Journal, (5), 171-180.

Winter, S. (2003). Understanding dynamic capabilities. Strategic management Journal, 24(10), 991-995.

Zollo, M., \& Winter, S. (2002). Deliberate learning and the evolution of dynamic capabilities. Organization science, 13(3), 339-351.

Cómo citar este capítulo:

Consuegra Ariza, I., Ariza Bethancourt, C., Barrios Hernández, K. (2017). Capacidad de innovación y gestión del conocimiento en una institución de educación superior. En K. Barrios Hernández, E. Olivero Vega, J. C. Acosta Prado, I. Meñaca Guerrero, A. M. Cazallo Antúnez, H. J. Medina Carrascal, . . O O. Hernández Ariza, Gestión del conocimiento y capacidad de innovación. Modelos, Sistemas y Aplicaciones (pp.251-280). Barranquilla: Universidad Simón Bolívar. 